\title{
Flexible design of commercial systems under market uncertainty: framework and application
}

\author{
Konstantinos C. Kalligeros* and Olivier de Weck ${ }^{\dagger}$ \\ Massachusetts Institute of Technology, Cambridge MA 02139
}

\begin{abstract}
A procedural framework is presented for the design optimization of modular commercial systems that are required to respond to changing market conditions. The basic premise is that commercial systems optimization aims directly at stakeholder value maximization. When stakeholder value is a quantifiable function of design variables and stochastic state variables, it can be maximized by maximizing expected value estimates plus the value from flexibility that stems from a design solution. We propose a multi-disciplinary framework, in which standard options valuation theory is used to model and valuate the flexibility embedded in a design solution, and external optimization techniques are used to determine the design of greatest value. We argue that the proposed framework is more intuitive and practical than equivalent stochastic optimization models. We demonstrate the framework in the preliminary design of the new Exploration Headquarters for British Petroleum in Aberdeen, Scotland. Preliminary results indicate that if flexibility is explicitly included in value-driven optimization, the resulting designs are quite different.
\end{abstract}

\section{Nomenclature}

$L \quad$ Stochastic value of land, $\$ / \mathrm{SF}$

$V \quad$ Stochastic intrinsic value of housing regional operations, \$

$v \quad$ Intrinsic value of office space, $\$ / \mathrm{SF}$

$\sigma_{L} \quad$ Annual volatility of land value

$\sigma_{V} \quad$ Annual volatility of regional operations

$d_{V} \quad$ Continuously compounded annual dividend rate of regional operations

$Y \quad$ Index of system configuration

$y \quad$ Index of modules

$C \quad$ Construction quality (normalized)

A Module area, $\mathrm{SF}$

$F \quad$ Number of floors per module

$\delta t \quad$ Time increment of the dynamic program, years

$r_{f} \quad$ Continuously compounded annual risk-free rate of return

$\bar{V} \quad$ The value of lost "capacity"

$P \quad$ Option payoff, $\$$

$\pi \quad$ Option value, $\$$

$K \quad$ Strike price, $\$$

$\rho \quad$ Correlation coefficient

$V_{s t} \quad$ "Static" value, determined based on expectations, $\$$

$V_{f l} \quad$ Value of flexibility, $\$$

$\Psi \quad$ Construction cost, $\$$

$V_{\text {tot }}$ Total value of a design solution, $\$$

\footnotetext{
${ }^{*}$ Graduate research assistant, Engineering Systems Division (ESD)
}

$\dagger$ Assistant Professor, Department of Aeronautics and Astronautics, Engineering Systems Division (ESD), Member 


\section{Introduction}

Through their financial success or failure, numerous system designs have illustrated the importance of accounting for design flexibility in the development and operations phase. Design flexibility provides developers and users with the ability to change a system's architecture, configuration and design variables so that it adapts to changes in its environment, both during development and operations.

Traditionally, the environmental changes engineers concern themselves about are those that affect safety, performance and reliability of a system. Numerous approaches have been developed to facilitate design subject to such uncertainties in the optimization and management science literature; a well-known example is the so-called six-sigma design. In contrast, we address changes that affect the financial performance of large scale engineering systems. Examples of such changes include price of oil, demand for a particular product, or technological innovations that affect the price or performance of critical system components. We seek to optimize systems to be flexible to respond to such changes. Typical responses include waiting to proceed or invest in subsequent development, expand (i.e., increase capacity), downsize (contract) the level of operations, completely abandon a project, switch between different inputs and/or outputs, or extend the service life of a system. ${ }^{1,2}$

The flexibility to respond to changes in any of the ways above is always valuable; sometimes, its value makes the difference between a system's ex-post financial success or failure. Even if the cost of, say, increasing capacity, is very high, the ability to do so is valuable if the operator of the system is not obliged to do so. Most of the time, the cost to respond to changes (e.g., by increasing production capacity) depends on initial design decisions. Different initial designs imply different costs to be paid in order to respond to environmental changes. Moreover, acquiring a lower switching cost between configurations may incur higher costs to build or operate in the initial or final configuration.

To stimulate intuition, consider the simple example of designing a manufacturing plant. Design solution $A$ is to build the capacity $c_{A}$ needed to fulfill current demand $d_{0}$, and in such a way so that expansion to $c>c_{A}$ is relatively costly. Another approach, call it $B$, is to build excess capacity $c_{B}-d_{0}$ to fulfill anticipated demand over an appropriate time horizon, and utilize only $d_{0}$ now. In $B$, increasing production up to $c_{B}$ is relatively cheap, but initial construction and operation costs will be higher than in $A$. Moreover, assume that building additional capacity $c-c_{B}$ is much more expensive than in $A$. Both designs offer the operator of the facility some flexibility to increase production; $A$ offers it at a higher switching cost, but lower construction and variable costs (for the initial capacity), while $B$ offers it at higher initial and variable costs, but a lower cost to switch to greater production scale. Finally, consider solution $C$, designed optimally given the expectation of future demand, but with infinite costs to increase it. If demand is certain to follow its expected path, then solution $C$ will be optimal by definition. However, uncertainty in the evolution of demand might make solutions $A$ or $B$ more appealing. The difference in value expected from $A$ or $B$ relative to $C$ in an uncertain environment is what we refer to as the value of flexibility embedded in a design solution. It is defined relative to the completely inflexible solution, $C$, and it is a function of the level of uncertainty in future projections.

From an optimization viewpoint, choosing between the three solutions is usually expressed as a valuemaximization, average cost-minimization or total cost-minimization problem, with a 3-point trade space. The constraints are simply the technical and physical laws that govern which designs are feasible (i.e., the feasible combinations of construction, variable and switching costs). Of course, in most engineering systems, the constraints will allow for a much more populated design space.

This example was fairly representative of the problem we will discuss in this paper; however, it is not original. Analyses of similar problems can be found in the so-called real options and capital budgeting literature, where the objective is the valuation of managerial flexibility available in projects. For the most part, the real options literature either focuses only on the valuation of flexibility (say, of designs $A$ or $B$ ), or uses stylized technical models to optimize design for flexibility in order to draw conclusions on economic implications. Examples include Triantis and Hodder, ${ }^{3}$ Kulatilaka, ${ }^{4}$ and Pindyck, ${ }^{5}$ to name only a few. Another, applications-oriented branch of the real options literature, takes care to be technically more accurate and realistic, and employs context-specific models for the valuation of flexibility in engineering or manufacturing systems. A few examples are from Dias, ${ }^{6}$ Zhao and Tseng, ${ }^{7}$ and de Weck et al, ${ }^{8}$

Related problems can also be dealt with using stochastic optimization tools that treat the valuation and optimization problem simultaneously. Examples include problems in production planning, capacity expansion, finance, water management and design of chemical processing units, among others. ${ }^{9}$ Research primarily focuses on efficient solution algorithms especially for linear and mixed-integer stochastic programs. ${ }^{10,11}$ 
We propose a framework that dissociates the valuation and optimization problems, and use real options analysis for the former and deterministic multi-disciplinary optimization tools for the latter. This approach is mathematically equivalent to existing stochastic optimization algorithms (see Wang and de Neufville ${ }^{12}$ ) while we believe it has two merits: First, it provides better insight into the design problem. Second, it explicitly relates technical considerations for new projects with financial objectives of large-scale systems, facilitating cross-disciplinary collaboration between engineers and managers within a firm. We demonstrate the framework in a preliminary model for the design of the new Exploration Headquarters for British Petroleum (BP) in Aberdeen, Scotland. We find that, even in a low-technology application such as an office complex, the optimal design is quite different when its embedded flexibility is accounted for. We close with a brief discussion of the approach.

\section{Proposed valuation/optimization procedure}

In a previous paper, ${ }^{13}$ we have shown that various academic efforts to quantify and optimize flexibility using real options implicitly use a common problem formulation. Here, we present a procedural framework for applying this formulation, summarized in the six steps described below.

1. Uncertainties identification and model In the simple example in the outset we demonstrated that the driver for embedding flexibility in a project is uncertainty. Project uncertainties need to be determined and modeled in such a way so that off-the-shelf options models can be used. In this paper, we use the approach described in Copeland and Antikarov. ${ }^{14}$

2. Architecture and design model The adaptability of engineering systems to changing conditions and their financial performance under expected conditions is a function of their architecture and design. To include flexibility for re-configuration in systems design, these architecture models need to be enriched with more information than what is typically used by engineers. The "design vector" must be such as to describe the system's configuration at the development or operations phase.

3. Project value model Here we bring steps (1) and (2) together; we model the financial value of a system design given in (2) for different levels of the uncertainties modeled in (1). From an economic standpoint, this step is easy and the theory is developed. In real life, it requires data and engineering models that are usually not readily available.

This step requires interdisciplinary input: the resulting value model must conform to the equilibrium assumptions on which options pricing theory is based, and this requires economic expertise. Secondly, mapping engineering quantities onto value or cash flows requires technical expertise.

4. Reconfiguration model A model of possible (and reasonable) re-configurations is needed, that can be described by the design vector in (2). The re-configuration model can be thought of as a network between point designs in the design space, in which each link is associated with a transition cost. The transition cost is a function of the design vector in (2) and possibly the uncertainties that affect the system. In options parlance, the re-configuration model determines the set of options available to the developers and operators of the system at any point in time.

5. Valuation of flexibility Step (5) is to assess the value of a system that allows (costly) transition between the operating modes described above. We use option valuation theory for this purpose. This is the value of flexibility over a system that can only operate at specified levels of its design variables. When a system can be reconfigured back and forth between two modes, path dependencies make option valuation conceptually challenging. Computationally, valuation is costly as it must be performed simultaneously for all feasible point designs.

6. Design space exploration and optimization Optimization is finally performed over the feasible initial designs of the system. The objective function is total value, which includes a static component (based on performance under expected conditions), a flexibility component (valued in step 4), and an initial development cost. Optimization is deterministic, but the trade-space is typically non-convex. ${ }^{10}$ 


\section{Application}

The conceptual framework presented above is demonstrated in the design optimization of the new British Petroleum (BP) Exploration Headquarters in Aberdeen, Scotland. The facilities are designed in response to BP's current demand in office space in the region and the obsolescence of the structure that houses its headquarters today. Instead of designing a conventional structure, the consulting architects (i.e., the Building Technology Laboratory at MIT) proposed a system of buildings that is flexible to gradually adapt to the company's declining space requirements.

This development project is somewhat unique; contracting operations is not usually a concern in the design of owner-occupied commercial office space located in central business districts: Commercial property and housing markets are usually "deep" enough so that owner-occupied office space can be leased out or sold when not needed. BP's exploration headquarters are probably one of the largest office campuses in a region with fast-rising demand for residential land and a very thin market for office space. ${ }^{\text {a }}$ Building a durable asset in such a market is probably not reasonable; it commits land to a use which is of high intrinsic value to its current owner, but of low value to the regional market. Leasing or buying existing space was apparently not a candidate solution because of the lack of a commercial campus of sufficient size in the region. BP thus decided to fund the building of a new facility, and the question of how to optimally design such a building arose.

\section{A. The model}

\section{Uncertainties identification and model}

As described in the previous section, the first step towards designing for flexibility is to identify the sources of uncertainty that give flexibility value. Based on the market conditions described previously, we seek to design a space that can be down-sized to the needs of its owner. BP's incentive to shrink the facility gradually is that it will realize benefits from converting the redundant space to a more profitable use. In an initial design proposal, the consulting architects suggested that conversion is designed as partial demolition and adaptive re-use. Therefore, the sources of uncertainty that might make a flexible design more valuable than an inflexible one, are

1. The value of land for residential use, on which the complex is built, $L(\$ / \mathrm{SF})$

2. The intrinsic value of the complex to $\mathrm{BP}, V(\$ / \mathrm{SF})$

Residential land value has been growing rapidly in the region over the past 10 years. ${ }^{16}$ Figure 1 shows residential land returns in Scotland from 1985 to 2004. ${ }^{\text {b }}$ Assuming the value of land follows a Geometric Brownian Motion (GBM), the data implies an annual volatility of $\sigma_{L}=14.82 \%$. ${ }^{\text {c }}$ Also, in 2002 (the starting date of our analysis), the value of land was $L(t=0)=12.82 \$ / \mathrm{SF}$.

At the same time, British Petroleum projections for the value of its regional operations are dramatic; the personnel numbers in Aberdeen fell from 3000 in 1999 to 2500 in 2001 and are expected to further decrease to about 1500 when the new building is completed in 2005. Officials admit that the company's operations ran from Aberdeen will have declined by $95 \%$ by 2015 at the earliest, and by 2045 the latest. Based on these projections, and treating $\mathrm{BP}$ regional operations as a dividend-paying project which is traded in the market, we followed the volatility estimation process described in Copeland and Antikarov ${ }^{14}$ to deduce an annual volatility of returns from the use of the building in the order of $\sigma_{V}=16.34 \%$, and a proportional annual dividend of $d_{V}=16.5 \%$. The calculations assumed that the project value also follows a GBM, an assumption which may also need future refinement.

\section{Architecture and design model}

Following uncertainty modeling, we needed a model of the system (i.e., the building) that completely characterized a design solution in terms of static value and reconfiguration costs. According to our design model,

\footnotetext{
${ }^{a}$ According to the PMR report $(2002)^{15}$ average rent for office space in Aberdeen was $26.5 \$ / \mathrm{SF} / \mathrm{yr}$ regardless of building quality or size.

${ }^{\mathrm{b}}$ Unfortunately, we were not able to find data specific to the Aberdeen land market.

${ }^{\mathrm{c}}$ The assumption of GBM for land value is not entirely correct, since the land itself is an option on the value of the best use that can be built on it. It is used here as a first approximation.
} 




Figure 1. Residential land returns in Scotland from 1985 to 2004

the office complex is comprised of $N$ adjacent modules, which can be converted, re-used or demolished independently in the future. Each module $y$ can be $F(y)$ floors high and have a gross square footage of $A(y)$. Finally, each module can be designed to different quality specifications, $Q(y)$. Design quality is further explained below. The vectors $\{A(y), F(y), Q(y)\}, y=1 \ldots N$ are enough to fully characterize the building complex design.

\section{Project value model}

In this application, linking the design vector to financial performance was relatively easy because of the abundance of approximate models and empirical data in the building industry and the real estate market. We developed the project value model by first quantifying the intrinsic value of office space to BP, based on the expectation for future operations. We then accounted for the effects of varying design quality and building obsolescence.

INTRINSIC VALUE, $v$ Intrinsic value can be thought of as the value of productivity of office space for the current occupant. If the owner's regional operations demand office space, then this space has intrinsic value; if they don't, then the space is redundant. This conceptualization is much like production capacity and demand, described in the example in the outset.

Assuming that BP would be willing to pay $26.5 \$ / \mathrm{SF} /$ year to support these operations, and knowing that the initial specification for square footage was $180,000 \mathrm{SF}$, we did a simple net-present-value analysis of the cash flows BP would be willing to commit to housing their declining regional operations (a proxy to rent). For discounting, we used the company stock's expected returns in the London stock market (ticker: BP.L). Based on that, we estimated the value of a facility that can house regional operations for the expected time horizon to be $V(t=0)=\$ 46,500,000$, or $v=\$ 258 / \mathrm{SF}$ for a certain level of design quality.

The value of regional operations $V$ is a stochastic variable; however, the intrinsic value of office space $v$ is not. At $t=0$, and given the preliminary proposed design of $180,000 \mathrm{SF}$, they are by definition equal: $V(t=0) \equiv 180,000 v$.

DESIGN QUALity, $Q$ Office space is built to various quality specifications. These depend on the quality and modularity of mechanical systems (such as heating, ventilation and air-conditioning), size, partitioning system, wiring, lighting and others. Detailed hedonic regression models of value (or rent) on factors such as these are really difficult to build for commercial property ${ }^{\mathrm{d}}$ because of the heavy dependence of value (and rent) on location. A rough classification for the UK on building characteristics alone, distinguishes between "type-1", "type-2" and "type-3" office space. This classification aggregates age, size, quality and building amenities. Type- 1 corresponds to large office complexes of top quality, while type-3 describes small, good

\footnotetext{
${ }^{\mathrm{d}}$ On the other hand, such a regression model for residential buildings is easier; see. ${ }^{17}$
} 
quality converted former Georgian or Victorian houses usually just off the town center. In "deep" real estate markets, where higher construction quality demands higher rent, the average yield difference between these type- 3 and type- 1 properties is of the order of $60 \%$. Figure 2 shows a regression on the rent levels demanded by each of the two types for selected, "deep" UK markets. ${ }^{15}$

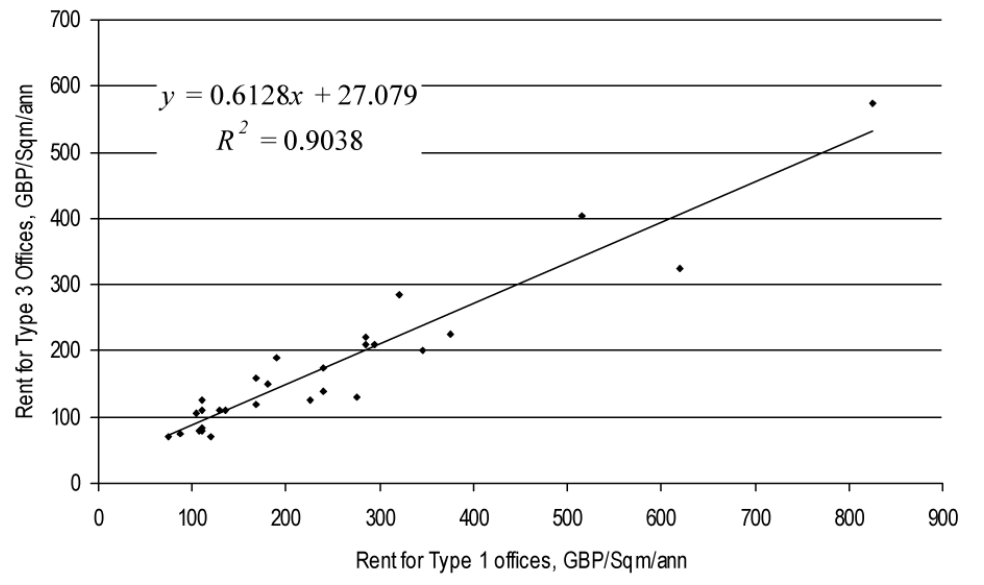

Figure 2. Contractual rent levels for type-1 and type-3 properties. Each point represents a particular market

Based on this data, we let $Q$ range between 0.833 and 1.200 . The rationale for the bounds is that new construction is never of comparable quality to type-3 space. The choice is mostly between exceptional $(Q=1.2)$, standard $(Q=1.0)$ and what is called in the US, "class-B office space" $(Q=0.833)$. The intrinsic value calculations in the previous section assumed that the initial design considered was of "standard" quality.

Design and construction quality and its effect on market rent in deep markets is relevant, because it partly reflects the increased productivity of high-quality office space when building stock and demand are in equilibrium. We implicitly assume here, that BP realizes productivity gains from using high-quality office space proportional to the ratio of rent levels regressed on the data in figure 2 .

BUILDING OBSOLESCENCE Buildings lose market value as their systems physically break down, require repairs, or as new technology renders building systems or architectural standards obsolete, and as the needs of end-users change. Iselin and Lemer ${ }^{18}$ and Lemer ${ }^{19}$ provide a qualitative explanation and model of obsolescence in buildings. The fact that buildings age is also evident from empirical data. Deterioration of physical property will gradually decrease the intrinsic value of a building to its owner, as it will decrease the building's market value.

We assume that the value of a building declines continuously at a rate $\theta \cdot{ }^{20}$ From aggregate data on office space across the UK, ${ }^{21}$ we were able to estimate the aging rate $\theta=1.02 \%$; see figure 3 . The number agrees with empirical data from interviews with US-based real estate brokers as well.

Inclusion of the aging effect in the model can be important, a priori at least. Since building value seems to decline exponentially, the value of buildings of lower initial value (e.g., because of lower construction quality) will decline slower than lower-quality buildings in absolute terms. In turn, this affects the economic value to convert, demolish or abandon a building. Intrinsic value over time and option value are thus coupled with construction quality, which is a direct design variable.

Construction cost, $\Psi$ Approximate functions of construction costs versus our design vector were built by combining preliminary neural network-based model results by Howard, Packer and Tate ${ }^{22}$ and normalized data from online resources such as RS Means. ${ }^{23}$ The former model relates gross square footage and number of floors to construction cost (figure 4), while with the latter we were able to extract linear factors for design quality. The result is a polynomial $\Psi=\Psi(A(y), F(y), Q(y))$ of $3^{\text {rd }}$ degree in $\mathrm{F}(\mathrm{y})$ and linear in $\mathrm{A}(\mathrm{y})$ and $\mathrm{Q}(\mathrm{y})$. 


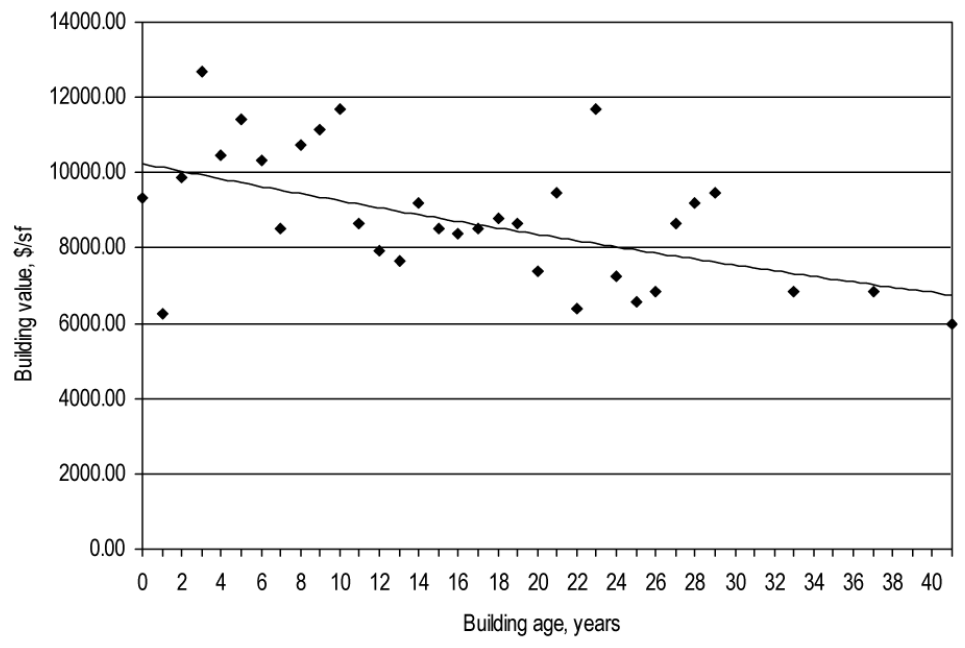

Figure 3. Aging of aggregate building stock in the UK



Figure 4. Construction cost model. The lines correspond to different total building area (in square meters)

\section{Reconfiguration model}

In the preliminary design proposition by the Building Technology Laboratory at MIT, the consulting architects proposed an 8-module design capable of two contraction modes. Figure 5 shows one of them, the "sequential" mode, which proceeds by abandoning and converting from one side of the building only. In reality, BP may follow the "sequential" contraction mode up to some point, and the proceed with other possible re-configuration sequences. In this paper, we only consider this sequence of possible reconfigurations to reduce computational burden, but examine a variable number of modules $N$, and how this affects total project value.

Finally, re-configuration costs need to be defined. Higher modules of larger area should reasonably incur higher hard costs to convert or adaptively re-use. For simplicity, and because of lack of relevant data, we neglected this dependence of re-configuration costs on design variables and assumed that hard reconfiguration costs are a flat proportion of the land area being freed up. These costs are thus included in the price of land. 


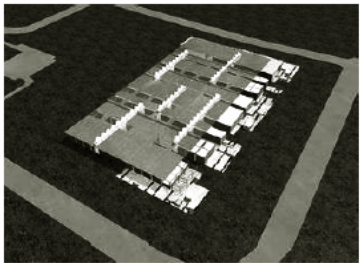

Phase 1

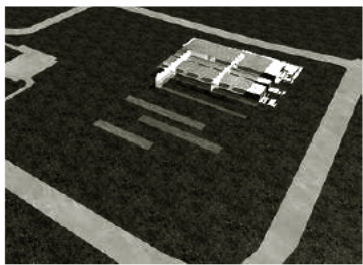

Phase 3

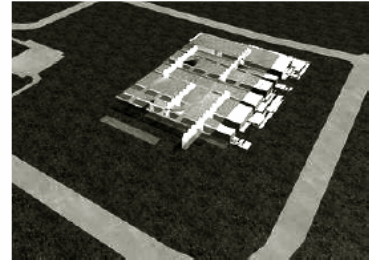

Phase 2

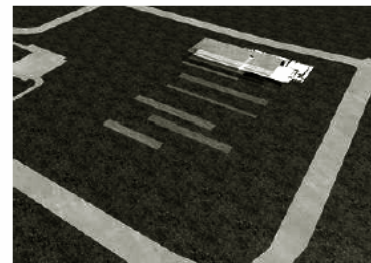

Phase 4

Figure 5. Rendering of the phased contraction of the office complex (courtesy: J. Fernandez)

\section{Valuation of flexibility}

In line with the conceptual framework presented in the previous section, we formulate the real options problem of valuing design flexibility as a function of the design variables and the architecture of the system. Given a modular architecture with $N$ modules, and the contraction sequence described above, we characterize the state of the system by the number of modules still in use, $Y(t) \in\{0,1 \ldots N\}$. At any point in time, BP has the option to switch from configuration $Y$ to $Y-1$ by abandoning module $y=Y$. ${ }^{\text {e }}$ In options parlance, this is referred to as a contraction option. It is an American-style put option on the value of lost capacity, with strike price the value of benefits gained from giving up capacity.

The value of flexibility embedded in a design of the entire system depends on the policy BP follows in abandoning modules. In turn, there exists an optimal policy for that. The trade-off's BP faces at any point in time are between abandoning the current configuration $Y$, or staying with it. The (expected) value of keeping the current configuration of the facility for one more time period reflects the value of having the "right, but not the obligation" to switch to the next configuration. Put simply, perhaps it will be optimal to switch to another configuration at the next time period. On the other hand, switching to configuration $Y-1$ at time $t$ generates a cash inflow corresponding to the value of land being freed up (a stochastic variable), and reveals an underlying option to subsequently switch from $Y-1$ to $Y-2$ at a later time. This option was not available directly with configuration $Y$. Switching also involves giving up on the value of the income-generating capacity of module $y=Y$ (also a stochastic variable).

To solve for the optimal policy, we first set up the discrete-time stochastic approximation of the stochastic processes $L$ and $V$ under the martingale measure according to standard contingent claims analysis. Details of the procedure can be found in standard option valuation textbooks; see Hull ${ }^{2}$ for example. Formally, land value and project value evolve under the equivalent martingale measure (EMM) according to equations (1) and (2) in discrete time, where $\delta t$ is the time increment used in the algorithm and $r_{f}$ is the risk-free discount rate.

$$
\begin{gathered}
L(t+1)=\left\{\begin{array}{c}
L(t) e^{\left(r_{f}-\sigma_{L}^{2} / 2\right) \delta t+\sigma_{L} \sqrt{\delta t}} \equiv V^{u}(t+1) \text { with probability } p=0.5 \\
L(t) e^{\left(r_{f}-\sigma_{L}^{2} / 2\right) \delta t-\sigma_{L} \sqrt{\delta t}} \equiv V^{d}(t+1) \text { with probability } 1-p=0.5
\end{array}\right. \\
V(t+1)=\left\{\begin{array}{c}
V(t) e^{\left(r_{f}-\sigma_{V}^{2} / 2\right) \delta t+\sigma_{V} \sqrt{\delta t}} \equiv V^{u}(t+1) \text { with probability } p=0.5 \\
V(t) e^{\left(r_{f}-\sigma_{V}^{2} / 2\right) \delta t-\sigma_{V} \sqrt{\delta t}} \equiv V^{d}(t+1) \text { with probability } 1-p=0.5
\end{array}\right.
\end{gathered}
$$

The value of "capacity" being given up by abandoning a module $y$ will depend on the value of operations, $V(t)$ and the intrinsic value of module $y$ to BP. Formally,

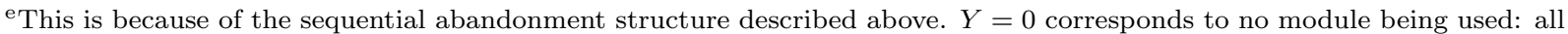
new modules have been abandoned, and whatever operations are housed in the existing facility, $y=0$. 


$$
\bar{V}(Y, t)=\max \left[0, \min \left[V(t), v e^{-\theta t} \sum_{y=0}^{Y} A(y) Q(y)\right]-v e^{-\theta t} \sum_{y=0}^{Y-1} A(y) Q(y)\right]
$$

where $\bar{V}(Y, t)$ is the value of capacity given up by switching to configuration $Y-1$, thereby giving up module $y$ at time $t$. Equation (3) says that, if the value of operations is above the capacity of configuration $Y$ to house them, then abandoning configuration $Y$ to obtain $Y-1$ gives up value $v e^{-\theta t} A(Y) Q(Y)$ only; if $V(t)$ is below $v e^{-\theta t} A(Y) Q(Y)$ but more than $v e^{-\theta t} \sum_{y=0}^{Y-1} A(y) Q(y)$ then the value of capacity being given up is $V(t)-v e^{-\theta t} \sum_{y=0}^{y-1} A(y) Q(y)$. Finally, if $V(t)$ is below $v e^{-\theta t} \sum_{y=0}^{Y-1} A(y) Q(y)$ then the value of lost capacity from abandoning configuration $Y$ is zero.

Therefore, the payoff of the option to contract from configuration $Y$ to configuration $Y-1$ is given by

$$
P(Y, V(t), L(t))=\max [0, K-\bar{V}(Y, t)]
$$

where $K$ is the "strike price" of the option, given by

$$
K(Y, t)=\frac{A(Y)}{F(Y)} L(t)+\pi(Y-1, V(t), L(t))
$$

where $\pi(Y, V(t), L(t))$ denotes the value (not the payoff) of holding configuration $Y$ when project value and land value are $V(t)$ and $L(t)$ respectively. Letting $\rho$ denote the correlation between $L$ and $V$, the expected value of holding $Y$ under the EMM is given by

$$
\begin{array}{rr}
E \pi(Y, t+1)=0.25[ & (1+\rho) \pi\left(Y, V^{u}(t+1), L^{u}(t+1)\right) \\
+ & (1+\rho) \pi\left(Y, V^{d}(t+1), L^{d}(t+1)\right) \\
+ & (1-\rho) \pi\left(Y, V^{u}(t+1), L^{d}(t+1)\right) \\
+ & (1-\rho) \pi\left(Y, V^{d}(t+1), L^{u}(t+1)\right)
\end{array}
$$

An appropriate discount rate needs to be used to discount $E \pi(Y, t+1)$ to time $t$, because the expectation is expressed with respect to information available at time $t$ and there exists risk in this expectation. Expressing the stochastic processes $L(t)$ and $V(t)$ under the equivalent martingale measure rather than their real probability measure, allows us to use the risk-free rate for discounting. This is the crux of what option valuation theory offers: it relieves the burden of appropriately choosing the discount rate to match the risk in the stochastic processes. For details, see Øksendal, ${ }^{24} \mathrm{Hull},{ }^{2}$ Copeland and Antikarov ${ }^{14}$ or Dixit and Pindyck. ${ }^{5}$

The optimal exercise policy at time $t$ proceeds according to the maximum value from either holding configuration $Y$ or switching to $Y-1$. The value of the option at $t$ will be

$$
\pi(Y, V(t), L(t))=\max \left[P(Y, V(t), L(t)), \frac{1}{1+r_{f}} E \pi(Y, t+1)\right]
$$

Equation 8 is Bellman's principle of optimality. Equations (1) to (8) can be solved using stochastic dynamic programming. In a quadranomial tree formulation, reflected in equations (1), (2) and (6), equations (3), (4) and (5) can easily be calculated at every node and for every configuration. Then, equation (8) is computed recursively, starting from all terminal nodes $(t=T)$ of configuration 0 , proceeding to time $t=0$ for $Y=0$, moving on to $t=T$ for $Y=1$ and repeating for $Y=2 \ldots N$. The value of flexibility embedded in the entire system is given by $\pi(N, V(t=0), L(t=0))$.

In parallel, the set

$$
X(Y) \equiv\left\{x=\{t, L(t), V(t)\} \mid P(Y, V(t), L(t)) \geq \frac{1}{1+r_{f}} E \pi(Y, t+1)\right\}
$$

is the locus of points (in our discrete time formulation) for which immediate conversion of module $Y$ is optimal. For each $Y$, these sets give the decision rules of the optimal policy. 
From a computational perspective, the quadranomial tree formulation is relatively slow for a reasonably small $\delta t$, which ensures satisfactory convergence. Alternative, more efficient models are needed for comparable convergence speed at lower computational cost (e.g., finite difference methods, see Tavella and Randal ${ }^{25}$ ). Nevertheless, with the framework proposed, use of off-the-shelf pricing tools is simple.

\section{Design space exploration and optimization}

The formulation presented in the previous section shows how different design solutions should give different values of flexibility, $\pi(N, V(t=0), L(t=0))$. Arguably, there exists a design that maximizes this value. However, the value of flexibility is not the only measure of value driving the design; according to our framework in section II, the optimal solution maximizes the sum of the value of flexibility, plus the "static" value of the system, $V_{s t}$. By the way the intrinsic value of a square foot of office space was quantified, $V_{s t}$ will simply be

$$
V_{s t}=\min \left[V(t=0), v \sum_{y=0}^{N} A(y) Q(y)\right]
$$

The total value of a design solution will then be $V_{t o t}=V_{s t}+V_{f l}-\Psi$. Intuition may be relatively easy for maximizing $V_{s t}$ or $V_{f l}$ separately, but unfortunately there is no clear intuition as to the design that maximizes $V_{t o t}$.

\section{B. Results}

We give preliminary space exploration results to demonstrate effects and interactions of the design variables, the effect of modularity, and the difference in design decisions if total value (versus static value) is the objective. In order to explore the trade space and assist intuition, we performed two experiment studies (a parameter study and a "one-at-a-time" set of experiments). The former involves designing experiments by sequentially using different levels for each design variable. The latter involves keeping the best of all designs evaluated up to each step, and experimenting with different levels for the remaining variables.

\section{Main effects of design variables}

Figure 6 shows the main effects of design variables, for the best solution found using the "one-at-a-time" set. The columns of plots correspond to value metrics, as described above. The last column includes construction costs. The plot rows correspond to design variables. Each bar in the sub-plots corresponds to a module.

The first thing to observe is that $V_{f l}$ is an order of magnitude lower than static value and construction cost. This is not because flexibility is not important; it is explained by the way $V_{s t}$ was defined. Recall the introduction, where we suggested that flexibility is only defined with respect to another solution. Static value already encompasses BP's flexibility not to use office space it does not need: $V_{s t}$ was calculated by decreasing the annual "rent" BP would be paying according to expectations for the value of operations. The additional value offered by the modular design reflects the firm's ability to benefit from conversion and re-use of land alone.

Figure (6) also shows that the main effect of $Q$ and $F$ differs among modules (compare panels d, e and f): the modules should not be built similar. For construction quality $C$, this is intuitive: modules expected to be used for less time than others should be designed at lower quality standards. Panels (g), (h) and (i) indicate that the number of floors should also vary between modules. Particularly, panel (h) indicates that the value of flexibility is maximized by building high expendable modules and low durable ones. Static value (panel g) however, demands high modules throughout the complex. Consideration of construction costs (panel i) indicates that total value is maximized for uniformly designing buildings around 5 storeys high.

Differentiation between modules in terms of gross area is not significant, according to panels (a), (b) and (c). The intuition is that there is a sufficient number of modules to allow the system to adapt to changing conditions "fast" enough. The intuition is confirmed if we compare figure 6 to panels (a), (b) and (c) in figure 7. With a total of three modules, the effect of module area becomes significant: the experiments indicate that at least the first module (i.e., the last to be converted) should be different than the others in terms of square footage.

Finally, notice how the design variables effect static value and value of flexibility differently throughout the columns in the two figures. Indeed, it seems, designing for flexibility results in different designs. 

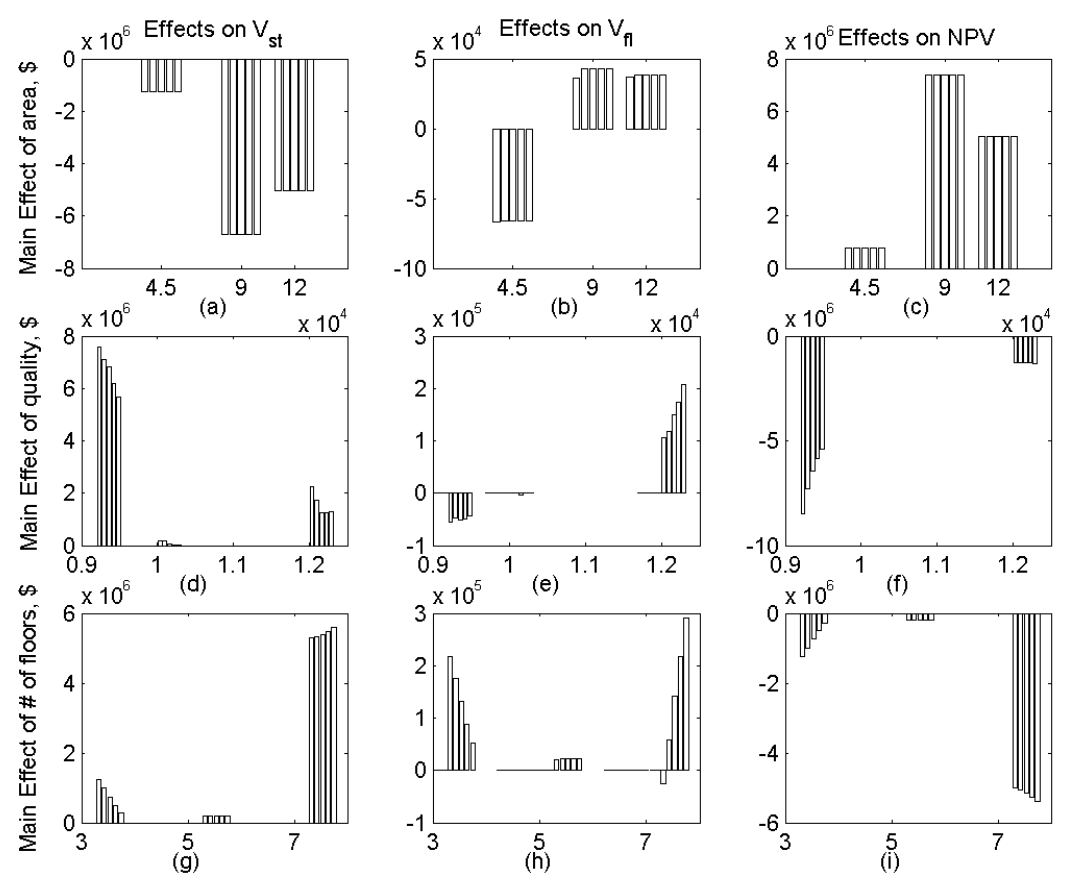

Figure 6. Main effects of design variables (rows) on static, option and total value (columns) for each of a total of 5 modules (bars).
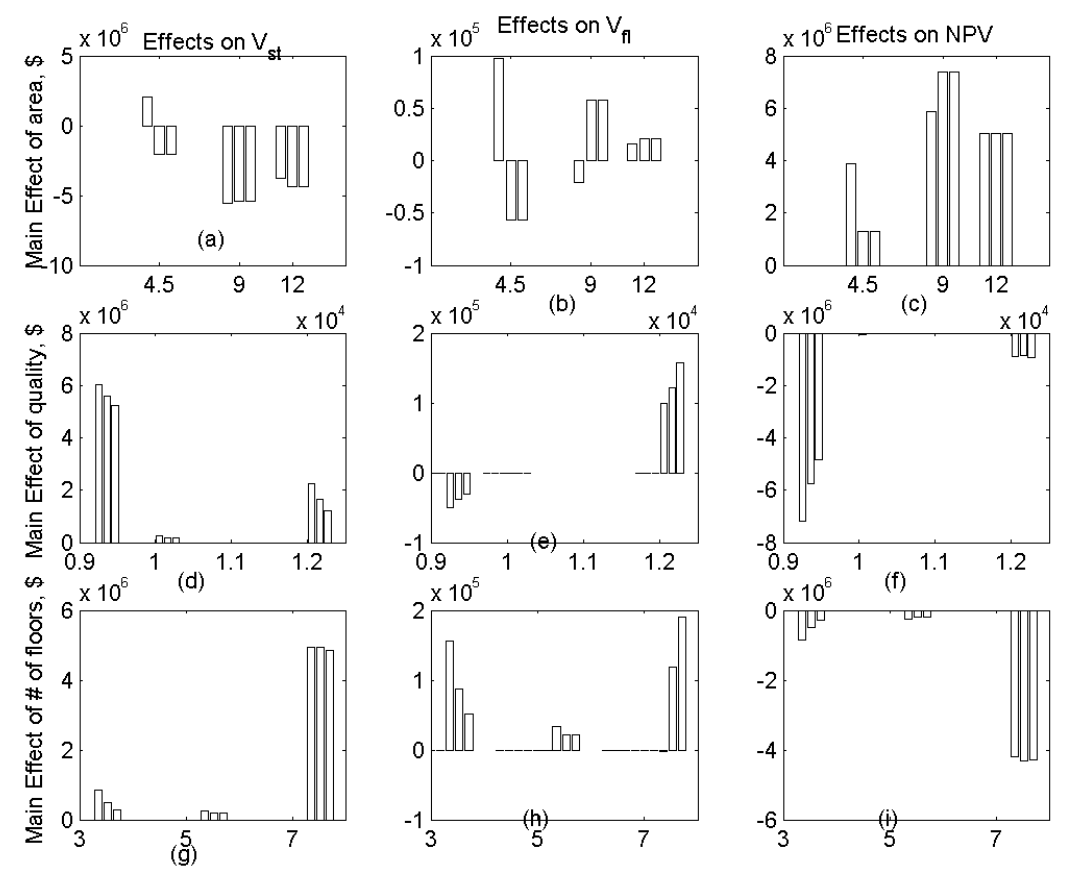

Figure 7. Main effects of design variables (rows) on static, option and total value (columns) for each of a total of 3 modules (bars). 

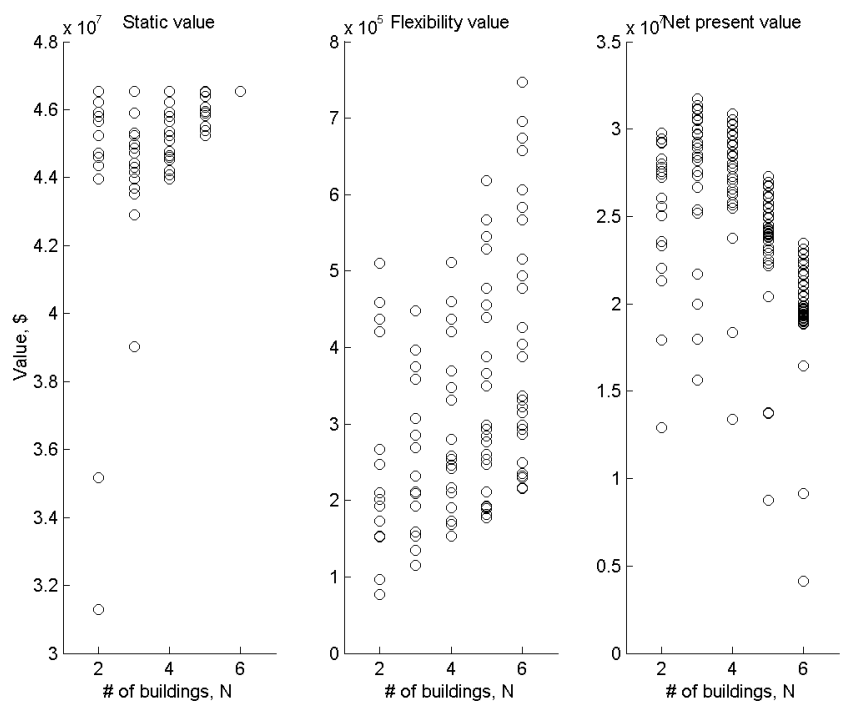

Figure 8. Effect of modularity: Each circle corresponds to an initial point design

\section{Effect of modularity}

Above, we hinted that the number of modules used, $N$, is significant for the effects of individual design variables. $N$ should also be significant for value objectives. In particular, we expect the value of flexibility to monotonically increase with the number of modules. The second panel in figure 8 confirms this. Static value (shown in the first panel), is naturally bounded above by the intrinsic value of the facility. If we consider construction costs and static value alone, the best design should probably be a single module with many floors. The third panel shows that flexibility causes an interior "optimal" solution with respect to $N$ : the best design in terms of total value has three modules. Such interior solutions are model-specific, not intuitive a priori, and can only be found using analytical models.


Figure 9. Decision surfaces for the best design of space exploration, with $N=3$

\section{Decision rules}

Finally, for the best experiment with $N=3$ (see figure 8), we extract the decision rules for conversion of each module (figure 9). They imply that conversion of module 3 is more "likely" to be optimal earlier than conversion of module 2 or 1 . The intuition is clear, but quantification of these optimal decision rules as of $t=0$ in accordance with the market's perception of risk in the project and value of land, requires the use of real options models. 


\title{
IV. Conclusion
}

We presented a real options -based framework for optimally configuring major commercial engineering systems with flexibility to adapt to changing conditions. It involves six procedural steps: Uncertainties identification; architecture and design modeling; project value modeling; a reconfiguration model; valuation of flexibility using option valuation theory; and external deterministic optimization. We demonstrated its use in the preliminary design of the new BP Exploration Headquarters in Scotland, and showed that designing with flexibility in mind results in quite different solutions than conventional, static approaches.

The framework presented can be applied to the design of a big range of engineering systems; nevertheless, further research is needed in all six steps in order to extract robust procedures and tools that can be widely used. Moreover, the framework's performance needs to be compared to alternative existing formulations (e.g., non-linear/integer stochastic programming) with respect to performance, intuition, applicability and ease of use.

We believe that when this framework matures, it will stand good chances of being adopted by the engineering community, as it explicitly relates technical considerations for new projects with financial objectives of large-scale systems, and it facilitates cross-disciplinary collaboration between engineers and managers within a firm.

\section{Acknowledgments}

We wish to thank Prof. John Fernandez at MIT for providing data and information on the BP project. We are also grateful to Prof. David Geltner, director of the Center for Real Estate at MIT for his insights and advice in earlier drafts of this work. Finally, we need to thank Prof. Roberto Pietroforte for sharing his construction experience and expertise.

\section{References}

\author{
${ }^{1}$ Bengtsson, J. Manufacturing flexibility and real options: A review. International Journal of Production Economics, 74, \\ 2001. \\ ${ }^{2}$ Hull, J., Options, futures and other derivatives, Fifth edition, Prentice Hall International editions, ISBN 81-203-2237-1, \\ 2000 \\ ${ }^{3}$ Triantis, A. and Hodder, J., Valuing flexibility as a complex option, Journal of Finance, 45(2):549-565, 1990 \\ ${ }^{4}$ Kulatilaka, N., The value of flexibility: the case of a dual-fuel industrial steam boiler, Financial Management, 22(3), \\ 1993 \\ ${ }^{5}$ Dixit, A. and Pindyck, R., Investment under uncertainty, Princeton University Press, New Jersey, 1994 \\ ${ }^{6}$ Dias, M., Selection of Alternatives of Investment in Information for Oilfield Development Using Evolutionary Real Options \\ Approach, Unpublished draft, 2001 \\ ${ }^{7}$ Zhao, T. and Tseng C. L., Valuing Flexibility in Infrastructure Expansion, Journal of Infrastructure Systems, September \\ 2003 \\ ${ }^{8}$ de Weck, O., de Neufville, R. and Chaize, M., Enhancing the economics of communications satellites via orbital recon- \\ figurations and staged deployment, American Institute of Aeronautics and Astronautics, AIAA-2003-6317, 2003 \\ ${ }^{9}$ Sahinidis, N. V. Optimization under uncertainty: state of the art and opportunities. Computers Chemical Engineering, \\ Accepted for publication, 2004. \\ ${ }^{10}$ Ahmed, S., King, A., and Parija, G. A multi-stage stochastic integer programming approach for capacity expansion under \\ uncertainty. Journal of Global Optimization, (26):324, 2003. \\ ${ }^{11}$ Ahmed, S. and Sahinidis, N. V. An approximation scheme for stochastic integer programs arising in capacity expansion. \\ Operations Research, 51(3):461471, 2003. \\ ${ }^{12}$ Wang, T. and de Neufville, R., Analyzing Infrastructure/Network Investments with Path-dependent Real Options, Pro- \\ ceedings, 8th Annual Real Options conference, Montreal, Canada, 2004 \\ ${ }^{13}$ Kalligeros, K., Using real options for engineering systems design: framework, applicability and limitations, Online pro- \\ ceedings, Engineering Systems Symposium, MIT 2004 http://esd.mit.edu/symposium/pdfs/papers/kalligeros.pdf \\ ${ }^{14}$ Copeland, T. and Antikarov, V., Real Options, A practitioner's guide, Texere LCC, New York, NY, 2001 \\ ${ }^{15}$ Valuation Office Agency, Property market Report for office space rent levels, Autumn 2002 \\ http://www.voa.gov.uk/publications/property_market_report/pmr_autumn_2002.htm \\ ${ }^{16}$ Valuation Office Agency, Property Market Report for land value, Autumn 2002 \\ http://www.voa.gov.uk/publications/property_market_report/pmr_autumn_2002.htm \\ ${ }^{17}$ Quigg, L., Empirical testing of real option pricing models, Journal of Finance, 48, 621-640 (1993) \\ ${ }^{18}$ Iselin, D. and Lemer, A.C. (editors), The Fourth Dimension in Building: Strategies for Minimizing Obsolescence, Studies \\ in Management and Building Technology, National Academy Press, Washington D.C. 1993
}


${ }^{19}$ Lemer, A., Infrastructure Obsolescence and Design Service Life, Journal of Infrastructure Systems, December 1996, pp. $153-161,1996$

${ }^{20}$ Geltner, D. and Miller, N. Commercial Real Estate Analysis and Investments, South-Western Publishing, Cincinnati, Ohio, 2001

${ }^{21}$ Office of the Deputy Prime Minister, Age of commercial and industrial property stock 2000, report, http://www.odpm.gov.uk/,2000

${ }^{22}$ James, H., Packer, A. and Tate, B., Determining the influence of design attributes on construction costs in COBRA 1995, ISBN 0-85406-739-6

${ }^{23} \mathrm{http}: / /$ www.rsmeans.com

${ }^{24} \varnothing \mathrm{ksendal}$, B., Stochastic Differential Equations: An introduction with applications, Springer-Verlag, New York, Berlin, Heidelberg, 1992

${ }^{25}$ Tavella, D., and Randall, C., Pricing Financial Instruments: The Finite Difference Method, J. Willey and Sons, New York, NY, 2000 